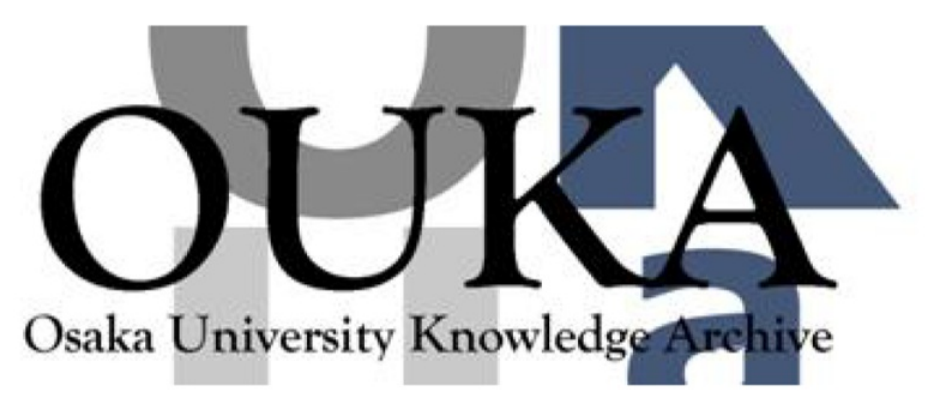

\begin{tabular}{|c|l|}
\hline Title & $\begin{array}{l}\text { Split-aperture laser pulse compressor design } \\
\text { tolerant to alignment and line-density } \\
\text { differences }\end{array}$ \\
\hline Author(s) & $\begin{array}{l}\text { Rushford, Michael C.; Britten, Jerald A.; } \\
\text { Barty, Christopher P..J. et al. }\end{array}$ \\
\hline Citation & Optics Letters. 33(16) p. 1902-p. 1904 \\
\hline Issue Date & 2008-08-15 \\
\hline oaire:version & VoR \\
\hline URL & https://hdl. handle.net/11094/3223 \\
\hline rights & \\
\hline Note & \\
\hline
\end{tabular}

Osaka University Knowledge Archive : OUKA

https://ir. Library. osaka-u. ac. jp/

Osaka University 


\title{
Split-aperture laser pulse compressor design tolerant to alignment and line-density differences
}

\author{
Michael C. Rushford, ${ }^{1, *}$ Jerald A. Britten, ${ }^{1}$ Christopher P. J. Barty, ${ }^{1}$ Takahisa Jitsuno, ${ }^{2}$ Kiminori Kondo, ${ }^{2,3,4}$ \\ Noriaki Miyanaga, ${ }^{2}$ Kazuo A. Tanaka, ${ }^{2,3}$ Ryosuke Kodama, ${ }^{2,3}$ and Guang $\mathrm{Xu}^{2,5}$ \\ ${ }^{1}$ Lawrence Livermore National Laboratory, 7000 East Avenue, Livermore, California 94550-9234, USA \\ ${ }^{2}$ Institute of Laser Engineering, Osaka University, 2-6 Yamada-oka, Suta, Osaka 565-0871, Japan \\ ${ }^{3}$ Graduate School of Engineering, Osaka University, 2-6 Yamada-oka, Suta, Osaka 565-0871, Japan \\ ${ }^{4}$ Present address: Kansai Photon Science Institute, Japan Atomic Energy Agency, 8-1 Umemidai, \\ Kizugawa-shi, Kyoto 619-0215, Japan \\ ${ }^{5}$ Present address: Shanghai Institute of Optics and Fine Mechanics, Chinese Academy of Sciences, \\ 390 Qinghe Road, Jiading, Shanghai 201800, China \\ *Corresponding author: rushford1@llnl.gov
}

Received April 9, 2008; revised June 2, 2008; accepted June 24, 2008; posted July 3, 2008 (Doc. ID 94873); published August 12, 2008

\begin{abstract}
We introduce a four-pass laser pulse compressor design based on two grating apertures with two gratings per aperture that is tolerant to some alignment errors and, importantly, to grating-to-grating period variations. Each half-beam samples each grating in a diamond-shaped compressor that is symmetric about a central bisecting plane. For any given grating, the two half-beams impinge on opposite sides of its surface normal. It is shown that the two split beams have no pointing difference from paired gratings with different periods. Furthermore, no phase shift between half-beams is incurred as long as the planes containing a grating line and the surface normal for each grating of the pair are parallel. For grating pairs satisfying this condition, gratings surfaces need not be on the same plane, as changes in the gap between the two can compensate to bring the beams back in phase. (C) 2008 Optical Society of America
\end{abstract}

OCIS codes: $050.1950,090.0090$.

Inertial confinement fusion short-pulse laser systems being built today [1-4] require arrayed diffraction gratings, given the current approximate meter-wide limitations of multilayer dielectric lamellar grating manufacture [4]. When tiling gratings coherently [3] a compressor focal spot with on-axis constructive interference is possible with intensities $>10^{20} \mathrm{~W} / \mathrm{cm}^{2}$ using kilojoule, picosecond pulses. However, achieving alignment of "identical" gratings to generate a constructive wavefront phase is challenging enough, and since gratings are manufactured serially, some small difference in the grating period between them is to be expected.

The LFEX compressor [5] being built at the Institute for Laser Engineering at Osaka University has a two-beam on-axis constructive interference, with two gratings spanning the beam aperture, even with linedensity difference between its four gratings. It is also tolerant to some degree of misalignment between the gratings. The beam size is $37 \mathrm{~cm} \times 37 \mathrm{~cm}$, and the average fluence is about $2.2 \mathrm{~J} / \mathrm{cm}^{2}$ on the incident beam. The element grating is a symmetrical lamellar-type multilayer dielectric reflection grating with a nominal groove density of 1740 lines $/ \mathrm{mm}$ across $91 \mathrm{~cm} \times 42 \mathrm{~cm}$. The gratings are being supplied by Plymouth Grating Laboratory [6].

Figure 1 shows the layout of the LFEX compressor, consisting of grating pairs G1A-G1B and G2A-G2B along with mirrors M1-M4. It depicts the chief ray paths for each half-beam, left (L) and right (R). Halfbeam L follows the path G1A, M1, G2B, M2, M3, G2A, M4, exiting G1B. Half-beam R follows the path G1B, M1, G2A, M2, M3, G2B, M4, exiting G1A. As can be seen, each half-beam hits each grating once, and for a given grating, each half-beam samples the grating from the opposite direction. Also, each beam is incident on consecutive gratings at the conjugate angle; i.e., the diffracted angle from one grating is the incident angle on the next grating. Using Fig. 1 as a reference, let us analyze the outcome if G1A has a slightly smaller period (larger line density) than the other three in an otherwise perfect compressor. For this discussion, we define $\alpha$ as the steep angle and $\beta$ as the shallow angle for the nominal diffraction conditions. Both angles can be incident or diffracted depending on the particular grating bounce being considered.

According the grating equation for the -1-order diffraction of half-beam L incident to G1A and adopting the convention that angles on the same side of a grating surface normal are positive,

$$
\sin (\alpha)+\sin (\beta)=\frac{\lambda}{p}
$$

the change in $\beta$ for beam $L$ owing to a perturbation in period $p$ of grating G1A is

$$
\mathrm{d} \beta_{\mathrm{G} 1 \mathrm{~A}}=-\frac{\lambda \mathrm{d} p_{\mathrm{G} 1 \mathrm{~A}}}{p^{2} \cos (\beta)} .
$$

For a decrease in period $(\mathrm{d} p<0)$, the angular perturbation is positive, as shown in Fig. 1. This angle is of course preserved upon the bounce from M1. Differentiating the grating equation for the perturbation in 


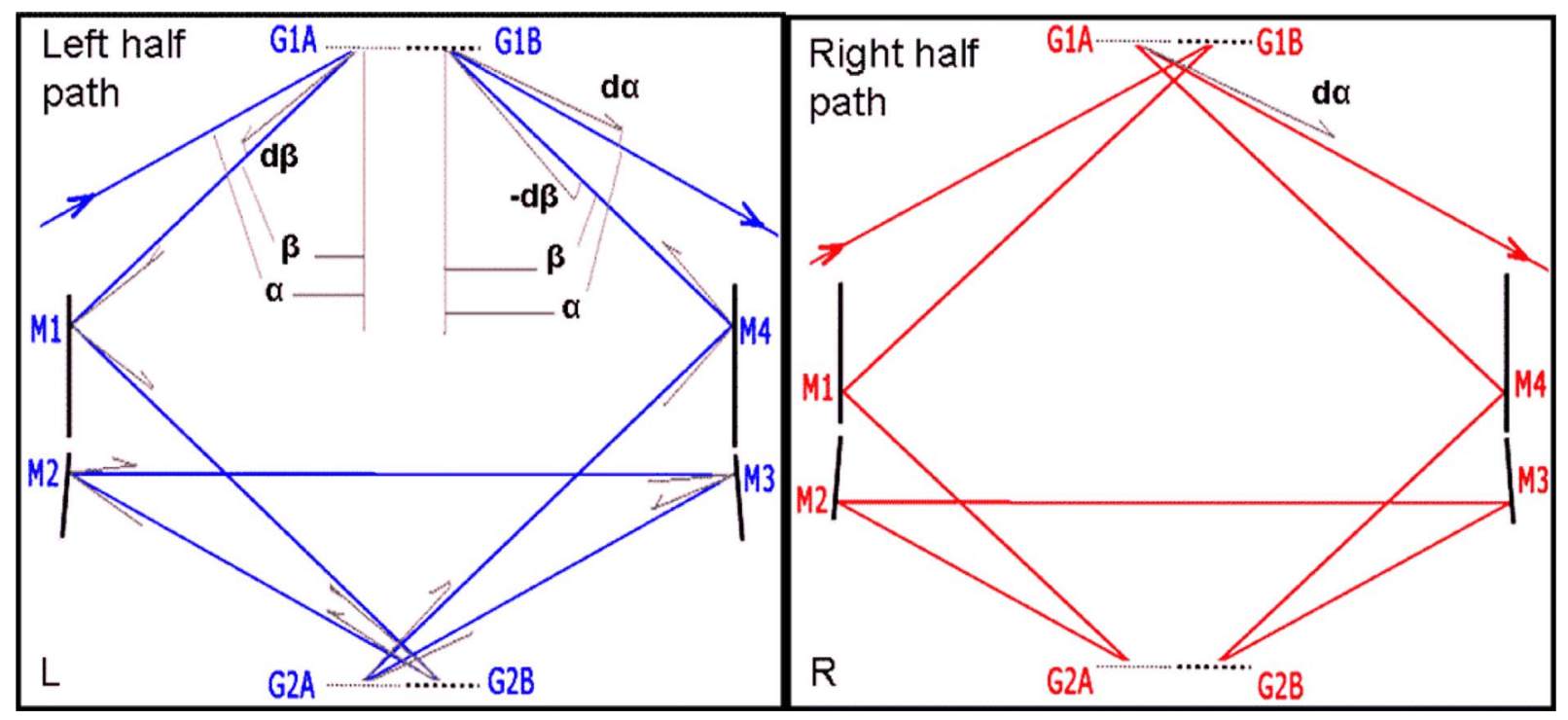

Fig. 1. (Color online) Schematic of the LFEX compressor showing principal left and right beam paths. The fletched gray arrows show the deflection angles of the beams if grating G1A has a grating period slightly decreased from the nominal value.

the incidence angle on the second grating beam $\mathrm{L}$ sees G2B:

$$
\mathrm{d} \alpha_{\mathrm{G} 2 \mathrm{~B}}=-\frac{\cos (\beta)}{\cos (\alpha)} \mathrm{d} \beta_{\mathrm{G} 1 \mathrm{~A}} .
$$

As beam $\mathrm{L}$ travels to the right side of the compressor via $\mathrm{M} 2$ and $\mathrm{M} 3$, the sign of the angle perturbation is flipped. Differentiating the grating equation again for angular perturbations upon diffraction of beam $\mathrm{L}$ from G2A, and using the result from Eq. (3), we arrive at

$$
\mathrm{d} \beta_{\mathrm{G} 2 \mathrm{~A}}=-\mathrm{d} \beta_{\mathrm{G} 1 \mathrm{~A}} .
$$

Differentiating the grating equation again for G1A, and using Eqs. (2) and (4), we arrive at

$$
\mathrm{d} \alpha_{\mathrm{G} 1 \mathrm{~A}}=-\frac{\lambda \mathrm{d} p_{\mathrm{G} 1 \mathrm{~A}}}{p^{2} \cos \left(\alpha_{\mathrm{G} 1 \mathrm{~B}}\right)} \text {. }
$$

For Beam R, differentiating Eq. (1) at G1A for a perturbation in period $\mathrm{d} p_{\mathrm{G} 1 \mathrm{~A}}$, we arrive at

$$
\mathrm{d} \alpha_{\mathrm{G} 1 \mathrm{~A}}=-\frac{\lambda \mathrm{d} p_{\mathrm{G} 1 \mathrm{~A}}}{p^{2} \cos \left(\alpha_{\mathrm{G} 1 \mathrm{~A}}\right)} .
$$

Since $\alpha_{\mathrm{G} 1 \mathrm{~A}}=\alpha_{\mathrm{G} 1 \mathrm{~B}}$, Eqs. (5) and (6) are identical. Thus both $\mathrm{L}$ and $\mathrm{R}$ beams exit the compressor on the same trajectory.

This analysis can be generalized to include the situation where all gratings have slightly different line densities. The exit angle of beams $L$ and $R$ are the same, but they are offset by a translation. For the LFEX compressor operating at $1053 \mathrm{~nm}$, the opticalpath difference (OPD) between $L$ and $R$ is one wave for a line-density change of G1A from 1740.0000 to 1740.0012 lines $/ \mathrm{mm}$. This line-density difference is about 0.7 parts per million, which is the expected accuracy of conventional grating fabrication. For this change, the pulse from $L$ and $R$ beam paths would have a group-delay difference of one wave. Any partial-wave OPD can be made constructive, yielding a high Strehl ratio focal spot, by adjusting the intergrating gap within a fraction of one grating period to compensate.

A tilt of one grating of a pair with respect to the other (a rotation where the grating surface normal stays in the plane of the paper of Fig. 1) causes the exiting $L$ and $R$ beams to diverge within the plane of the compressor. This can be understood by considering that since the two beams incident on one grating come in at their respective conjugate angles, a rotation will bring each either closer or farther from its Littrow angle. A rotation of a grating face around its surface normal will cause the beams to diverge with the opposite sign out of the plane of the paper. A tip of a grating (rotation with the grating surface normal in a plane orthogonal to the paper in Fig. 1) causes the beams to steer equally for $L$ and $R$ out of the plane of the paper. Since each beam half-wave is steered equally, only the wavefront piston phase needs adjustment, again by changing gap between gratings within one grating period. This leads to a fundamental property of this compressor: If gratings in a pair are oriented such that their planes containing a grating line and the surface normal are parallel, the beams exiting the compressor suffer no pointing difference. The gratings can be translated or rotated in any direction that maintains this condition without a pointing difference between the combined beams. Since each beam half-wave is affected equally, only the wavefront piston phase needs adjustment, again by changing the gap between gratings within one grating period. The grating faces need not be in the same plane. A small diameter beam collinear to the main beam path, which spans the grating gap, can be used to adjust tilt, in-plane rotation, and gap spacing to minimize the piston-phase difference.

Grating-to-grating gaps, inherent to tiled grating systems, will cause temporal and spatial pedestal at 
the focal plane [7]. The gap between the beams diffracting from grating pairs $\mathrm{G} 1 \mathrm{~A}-\mathrm{G} 1 \mathrm{~B}$ and $\mathrm{G} 2 \mathrm{~A}-\mathrm{G} 2 \mathrm{~B}$ should be less than $10 \mathrm{~mm}$. This gap comprises the physical spacing between grating edges, twice the grating bevel, and twice the width of the zone on the substrate where edge effects prevent the patterning of high-efficiency grating lines. Recently, Lauerence Livermore National Laboratory (LLNL) demonstrated high-efficiency grating lines within $250 \mu \mathrm{m}$ from the inner edge of the substrate chamfer on large-aperture gratings [8].

To summarize, the LFEX pulse compressor described here uses half of the gratings required in a conventional four-pass, two-beam, split-aperture compressor, and is tolerant to small line-density variations between gratings. It is also tolerant to tip alignment, and the orthogonal errors caused by tilt and in-plane grating rotation can be monitored by a probe laser spanning the gap between gratings in a pair. This gap can be adjusted to overlap the phase of the combined beams exiting the compressor. An experimental demonstration of this compressor type will appear in a forthcoming publication.

Portions of this work were performed under the auspices of the U.S. Department of Energy by Lawrence Livermore National Laboratory under contract DE-AC52-07NA27344. G. Xu acknowledges the support by Japan Society for the Promotion of Science, core to core university program 15 A: Study of Ultrahigh Density Plasma operated by National Institute of Fusion Science.

\section{References}

1. T. Zhang, M. Yonemura, and Y. Kato, Fusion Eng. Des. 44, 127 (1999).

2. T. Zhang, M. Yonemura, and Y. Kato, Opt. Commun. 145, 367 (1998).

3. T. J. Kessler, J. Bunkenburg, H. Huang, A. Kozlov, and D. Meyerhofer, Opt. Lett. 29, 635 (2004).

4. J. D. Zuegel, S. Borneis, C. Barty, B. Le Garrec, C. Danson, N. Miyanaga, P. K. Rambo, C. Le Blanc, T. J. Kessler, A. W. Schmid, L. J. Waxer, J. H. Kelly, B. Kruschwitz, R. Jungquist, E. Moses, J. Britten, I. Jovanovic, J. Dawson, and N. Blanchot, Fusion Sci. Technol. 49, 453 (2006).

5. N. Miyanaga, H. Azechi, K. A. Tanaka, T. Kanabe, T. Jitsuno, J. Kawanaka, Y. Fujimoto, R. Kodama, H. Shiraga, K. Kondo, K. Tsubakimoto, H. Habara, J. Lu, G. Xu, N. Mori, S. Matsuo, E. Miyaji, Y. Kawakami, Y. Izawa, and K. Mima, J. Phys. IV 133, 81 (2006).

6. http://www.plymouthgrating.com/.

7. Y. Hu (Tsinghua University, Beijing, China) is preparing a paper to be called "Effect of misalignment of tiled-grating on spatial and time-domain performances in dual-side-incidence pulse compressor."

8. Collaboration (https://www.llnl.gov/str/Verdon.html) on LLNL gratings delivered to Laboratory for Laser Energetics, University of Rochester, contact: britten1@llnl.gov. 